\title{
Ruptures et retournements de la sémiologie des médias à l'ère de la communication
}

\author{
François Jost ${ }^{\mathbf{1}}$ \\ Sorbonne Nouvelle Paris III
}

\begin{abstract}
Resumo: Quais as contribuições das ciências da comunicação para a semiologia? Com intuito de responder a esta questão, parto de duas experiências televisivas impossíveis de serem pensadas dentro do quadro de referência de uma semiologia imanente e sugiro uma reversão coperniciana. Para analisar a televisão, não é mais possível partir da imagem, deve-se partir dos gêneros. A partir daí, exponho quatro proposições que fundamentam meu sistema conceitual, que envolve uma nova concepção dos gêneros e da comunicação televisiva. Demonstro, ao mesmo tempo, que a "teoria do contrato" se refere apenas a um caso particular, o da "leitura escolada", sendo incapaz de pensar a relação dinâmica entre emissor $e$ receptor devido à promessa pragmática. O método exposto aqui é, em seguida, estendido ao campo cinematográfico, que demanda uma mudança epistemológica do mesmo tipo.
\end{abstract}

Palavras-Chave: televisão; semiologia; gênero; campo cinematográfico.

\footnotetext{
1 FRANCOIS JOST est professeur à la Sorbonne Nouvelle Paris III, où il dirige le Centre d'Etudes sur l'Image et le Son Médiatiques (CEISME) et où il enseigne l'analyse de la télévision et la sémiologie audiovisuelle. Il est Professeur invité dans de nombreuses universités à travers le monde, notamment au Brésil. Spécialiste de l'image, il a écrit de nombreux ouvrages sur le cinéma, dont L'Oeil-caméra (Presses Universitaires de Lyon, 1987, 2 éd. 1989), Le Recit cinématographique (avec A.Gaudreault, Paris, Nathan, 1990), Un Monde à notre image (Méridiens Klincksieck, 1993), Le Temps d'un regard. De l'image au spectateur (Méridiens Klincksieck, 1998). Il a contribué, ces dernières années, à développer en France les études théoriques sur la télévision, par la direction de numéros de revues, de colloques (Penser la télévision) et la publications de livres: Introduction à l'analyse de la télévision (Ellipses, 1999), La télévision du quotidien (De Boeck, 2001), L'Empire du loft (ed. La Dispute, 2002), Realtà/Finzione (Milan, Castoro), Seis lições sobre televisão (Porto Alegre, Editora Sulina, 2004), Années 70: la télévision en jeu, F. Jost ed., (CNRS ed., 2005), Comprendre la télévision, (Armand Colin, coll. 128, 2005). Scénariste et réalisateur, entre 1977 et 1987, il a écrit plusieurs programmes pour la télévision. Il a réalisé plusieurs films dont l'un, La Mort du révolutionnaire, hallucinée, a reçu trois prix (Festival international du Jeune cinéma, Hyères et Belfort, 1979). Il est aussi auteur d'un roman, Les Thermes de Stabies (MK Littérature, 1990).
} 
Résumé: Qu'ont apporté les sciences de la communication à la sémiologie ? Pour répondre à cette question, l'auteur part de deux expériences télévisuelles impossibles à penser dans le cadre d'une sémiologie immanente et suggère un retournement copernicien. Pour analyser la télévision, il n'est plus possible de partir de l'image, il faut partir des genres. À partir de là, il expose les quatre propositions qui fondent son système conceptuel, qui engage une nouvelle conception des genres et de la communication télévisuelle. Il montre du même coup que la théorie du " contrat » n'est qu'un cas particulier, celui de la lecture " savante », mais est incapable de penser la relation dynamique qui unit émetteur et récepteur au travers la promesse pragmatique. La méthode ici exposée est ensuite étendu vers le champ cinématographique, qui appelle un changement méthodologique du même type.

Mots-clé: télévision; sémiologie; genre; champ cinématographique.

Abstract: What brought communication sciences to semiology? To answer this question, the author analyzes two television sequences impossible to think within the framework of an immanent semiology and suggests a copernican reversal. For analyzing television, the starting point must not be image, but genres. Hence, Jost exposes the four propositions on which his conceptual system is based. They involve a new conception of genres and of TV communication. At the same time the theory of "contract" becomes a particular case, that of the "learned" reading, incapable to deal with the dynamic relation which links emitting and receptor because of the pragmatic promise. Then, the here explained method is exported towards the film field, which demands such a methodological change of paradigm.

Keywords: television; semiology; genre; film field.

On le rappelle assez souvent: la sémiologie appartient aux disciplines fondatrices des sciences de l'information/communication. Affirmant cette appartenance, la plupart des chercheurs s'entendent, encore aujourd'hui, sur un nom : Barthes, qu'ils annexent parfois, purement et simplement, à la sociologie des médias ${ }^{2}$. Pourtant, quand on considère la place qui lui est accordée dans les cursus de communication, la sémiologie des médias n'est pas loin de partager le sort des langues mortes dans ceux de Lettres modernes, celui d'une espèce en voie de disparition ${ }^{3}$. Si l'on considère à présent les objets sur lesquels elle porte son regard, sa position n'est guère plus favorable : au sein de la communauté «info-comm », la télévision apparait très généralement comme un objet vieillot, ancien, et l'on préfère

\footnotetext{
${ }^{2}$ Très symptomatique de cette attitude un récent ouvrage intitulé Sociologie des médias qui réduit la place de la sémiologie à une demie-page et ignore tout des recherches sur la télévision.

${ }^{3}$ Un exemple significatif : l'UFR Communication de Paris III a perdu dans les cinq dernières années pas moins de 4 postes de Maître de conférences ou de Professeur de sémiologie. On espère que c'est un cas particulier...
} 
parler de TIC, de téléphones portables ou de réseaux numériques, comme si tous ces nouveaux «tuyaux » avaient une vie indépendante. Quant au cinéma, c'est à peine si on lui reconnaît un droit de cité : depuis que le CNU a rendu autonomes les sciences de l'information et de la communication et que le cinéma a été rattaché le cinéma à l'Esthétique, pour être admis à parler de cinéma en $71^{\mathrm{e}}$ section (Sc. info/comm.), il faut donner des gages de son approche communicationnelle ou s'intéresser à des objets que l'esthétique ignore (publics, promotion, etc.).

Cette rapide entrée en matière semblera peut-être excessivement schématique, voire un tantinet pessimiste, mais je crois qu'elle résume au mieux la réponse que je peux faire à la question qui m'est posée : Qu'ont retenu les sciences de l'information / communication des théories sémiotiques sur les médias notamment ceux du cinéma et de la télévision ? J'aurais même pu faire plus court en répondant: pas grand-chose...

En revanche, si on prend les choses par l'autre bout et que l'on se demande ce qu'ont apporté les sciences de l'information-communication à la sémiologie du cinéma, il y a beaucoup à dire et le rôle de ce cet ancrage sur le développement de la discipline est indéniable. C'est donc de là que je partirai.

\section{De l'image aux genres}

Pour comprendre la situation actuelle de la sémiologie, il faut d'abord dégager quelques présupposés sur lesquels se fonde la première sémiologie du cinéma, celle de C. Metz. En premier lieu, toutes les travaux sémiologiques, qu’il s'agisse de ceux qui concernent le récit ou de ceux qui concernent le cinéma, prennent naissance dans un contexte où la « Communication » constitue à la fois une rupture de paradigme et une rupture avec les disciplines universitaires : le Centre d'Études des Communications de Masse, à l'École des Hautes Études en Sciences Sociales et la revue Communications regroupent des chercheurs qui n'ont pas toujours les diplômes requis par la carrière universitaire (Barthes n'a pas de thèse), et qui rompent avec les approches de la Sorbonne (cf. Critique et vérité et la querelle Picard-Barthes). La linguistique est le parangon de la scientificité pour la sémiologie naissante, en sorte que considérer le texte comme un message et l'image comme un texte sont des actes militants qui font consensus : qu'il s'agisse de photographie, de 
publicité ou de film, tout devient message. Si cette position a pour vertu non négligeable d'ouvrir l'éventail des objets d'étude possibles (la $\mathrm{BD}$, le roman policier, le gag, etc) et d'écarter a priori l'idée qu'il y ait de "mauvais objets », elle a pour revers une mise entre parenthèses de tout ce qui a trait au contexte, au sens très large qu'il va nous falloir expliciter.

Pendant toute une période, les chercheurs ont étudié les émissions de télévision comme si c'était des films ou comme si c'était des objets langagiers. Dans les deux cas, il s'agissait de transposer des méthodes qui avaient fait leurs preuves ailleurs, soit qu'on envisageât le montage ou le cadrage comme dans un film, soit qu'on réduisît la relation de l'émetteur et du récepteur à un «contrat de communication », comme dans une conversation. Pour un sémiologue comme moi, la lecture de La pertinence, de Sperber et Wilson, et La Transfiguration du banal, d'Arthur Danto, m'a installé définitivement dans l'ère du soupçon qu'avaient ouverte les philosophes du langage. Le rapprochement de ces auteurs peut paraître étrange tant leurs objets et leurs méthodes diffèrent, pourtant je tire des uns et des autres une même leçon. Qu'il s'agisse, comme les premiers, de démontrer que la communication verbale se fonde non sur le code, mais sur des inférences entre deux instances qui partagent plus ou moins un environnement cognitif, ou, comme le second, qu'une œuvre artistique ne se réduit pas à la matérialité de son objet, les bases de la sémiologie de l'image se trouvent ébranlées. Si les qualités matérielles d'un objet, énoncé ou boîte de conserve, importent moins que leurs usagers ou que le statut qu'on leur donne, comment, en effet, conserver les grandes catégorisations de l'image qui trouvent leur origine dans des propriétés objectivables, telles que images fixes (photo, dessin, peinture) vs images animées (cinéma, télévision, dessin animé), image unique vs succession d'images, images manuelles vs images dupliquées mécaniquement, etc. ? Cette question s'est posée pour moi par deux fois, avec acuité, lors de la retransmission de la révolution roumaine de 1989 (cf. Jost 1992).

La première fut, non pas l'image du charnier de Timisoara, dont on nous a rebattu les oreilles, mais ce moment, beaucoup moins remarqué, où pendant le procès expéditif du procès du couple Ceaucescu, l'image se "gelait », sans que les journalistes n’y prêtent attention : à espaces réguliers, le dictateur a sa femme apparaissaient sous la forme d'un simple instantané figé. En termes de sémiologie 
immanentiste, on aurait volontiers affirmé, avec Gaudreault que, dans ces moments, on se trouvait en deçà du récit, dans la mesure où «l'on ne saurait les considérer [l'arrêt sur image ou le plan immobile] comme des énoncés narratifs » (1988: 47). Or, replacés dans leur contexte communicationnel, où un émetteur cherche à dire quelque chose au travers des images, ces « frozen shots » apparaissaient comme de véritables actes de récit dans la mesure où ils visaient à censurer l'image des juges et, donc, à opérer une sélection sévère dans le réel et le visible. Du même coup, la nature de l'image en tant que telle (fixe vs animée) devenait moins signifiante que le renvoi à sa source et que la construction de l'intention qu'on pouvait en inférer.

Un autre événement télévisuel, chronologiquement antérieur de quelques heures, incitait à remettre en cause le paradigme structural, la retransmission « en direct » des événements de Bucarest. Soudain, alors qu'on voyait des insurgés traverser calmement un square, le drapeau national à la main, amputé des signes distinctifs du communisme, de la neige cathodique envahissait l'écran, le temps d'un changement de plan. De façon tout à fait étonnante pour moi, ce détail ne frappa pas le journaliste en plateau, qui attribua cet incident aux « aléas » du direct. Ce n'est pas que la «neige» cathodique interrompe un direct qui était remarquable, bien entendu, mais le fait qu'elle intervînt entre deux plans aux axes différents. Comment, au cœur de cette Histoire en devenir, le réalisateur avait-il pu disposer de deux caméras et d'un camion de régie pour mixer les plans? Pour attribuer cette imperfection visuelle au direct, il fallait supposer des moyens techniques totalement incongrus en la circonstance. Démarche qu'adoptait implicitement le journaliste.

L'analyse de l'image révéla que la réalité était tout autre : un caméraman avait enregistré l'avancée des insurgés dans un parc sous un angle donné, couru un peu plus loin et pris la scène sous un autre angle, arrêtant la caméra entre les deux prises, ce qui provoque un effet de neige cathodique. Du même coup, plusieurs présupposés de la sémiologie du cinéma s'en trouvait ébranlés : 1. Le fait qu'il faille partir des images isolées pour comprendre leur signification ; 2. Que le sens vienne de l'analogie ; 3. Que l'image ne soit que signe du monde (et non pas de source) ; 4. Qu'il soit possible de se fier au «contrat » que propose l'émetteur, à savoir icile direct. Autant de propositions qui fondent implicitement la sémiologie metzienne. 
Ces deux exemples imposaient ni plus ni moins qu'un retournement copernicien. Si dans le paradigme jakobsonien, on pouvait partir de l'image, soucieux de construire la «coupure sémiotique » d'avec le monde, qui la faisait accéder au statut de signe, dans un modèle de la communication non transitif, c'est-à-dire dans un modèle où le document n'est pas forcément à l'arrivée, pour le récepteur, ce qu'il était au départ pour l'émetteur, et où les inférences comptent tout autant que les codes, il faut, à l'inverse, poser que c'est le contexte qui donne le sens aux signes. D’où la nécessité de penser non plus l'image et le récit en général, mais l’image et le récit médiatiques. Ce qui impose, pour une succession de plans aussi simples que ceux du square de Bucarest, de ne pas étudier le changement de plan, pour lui-même - qui est un peu signifiant en tant que tel : Metz dirait que, du point de vue syntagmatique, c'est une "scène » -, mais de le corréler à plusieurs niveaux contextuels : 1. Le statut de l'objet audiovisuel : selon qu'il s'agit d'un direct ou d'une œuvre de fiction, voire d'une œuvre, le sens ne sera pas le même ; 2. Le genre, en l'occurrence, le direct, avec la question qu'il suscite : qu'est-ce que le direct et est-il compatible avec le montage des deux plans ? (en l'occurrence la réponse est non) ; 3. Le média : l'inscription «direct » dans un coin de l'image prescrit une croyance en l'authenticité des images. Faut-il pour autant aligner la réception sur les visées de l'émetteur, qui sont ici de renforcer cette croyance? Faut-il, en d'autres termes, accepter le modèle du « contrat » pour décrire la relation communicationnelle entre émetteur et récepteur ?4 Là encore, la réponse est non. Cet exemple révèle un cas typique de mensonge. D'où la conclusion que l'étiquetage générique n'est qu'une «promesse pragmatique » que le téléspectateur aurait tort de prendre toujours à la lettre.

On le voit : dans cette perspective, si l'on part de l'image, on n'y reste pas. On pose, au contraire, dans une optique communicationnelle, qu'il n'y a pas d'image qui ne soit replacée, pour être sémantisée ou interprétée, dans un processus d'induction qui élargit son horizon, d'attente ou d'interprétation. En cela, la télévision avec son

\footnotetext{
${ }^{4}$ P. Charaudeau lie en effet le «contrat de communication » à la visée du locuteur définie ainsi : «Dans la communication langagière, le but ne peut être que de tenter de faire entrer l'autre dans sa propre intentionnalité » (1997:69), ce qui, notons-le en passant, est bien loin du modèle de la communication verbale proposé par Sperber et Wilson, qui repose sur l'idée que les interlocuteurs visent à augmenter leur connaissance commune du monde.
} 
zapping est un cas de figure emblématique. Qu'on prenne une émission au hasard en rentrant tard chez soi, sur une chaîne qu'on connaît à peine, ou qu'on pousse le bouton du poste de télévision de sa chambre d'hôtel après avoir franchi de nombreux fuseaux horaires, la question est la même : à quoi dois-je rattacher les images que je découvre et que je ne connais pas ? Du côté de la production et de la programmation, l’interrogation est symétrique : pour comprendre ce qu'aiment les téléspectateurs, comment s'y retrouver dans le foisonnement forcément indéfinis des genres. Une étude récente de la NHK aboutit par exemple, à un classement de 547 émissions diffusées sur la chaîne en 49 genres et sous-genres. Comment déceler des tendances de réception dans un panorama aussi vaste? En l'occurrence, les auteurs de l'étude aboutissent à la constitution de grands axes : more/less serious et more/less fictional (Hara et al. 2004) Si ma réponse n'est pas la même, l'interrogation est du même ordre. Comment ramener la pluralité des genres à des archigenres qui permettent de comprendre leur engendrement et leur réception ou, en termes peirciens, quels sont les interprétants permettant de donner du sens aux images ? Dans la mesure où je tenais pour acquis que le cadre devait être communicationnel, la contrainte théorique était double : trouver un terrain d'analyse commun à l'émetteur, au récepteur et au document lui-même et faire en sorte que ce cadre ne postule pas un modèle de communication pacifiée, qui alignait forcément la réception sur les visées de l'émetteur.

Mon système conceptuel va dès lors se fonder sur quatre propositions :

1. L'ensemble des genres et des programmes de télévision peut être catégorisé en fonction de trois mondes, qui jouent en quelque sorte le rôle d'archigenre ou, en termes peirciens, d’interprétant.

2. Tout genre repose sur la promesse d'une relation à un monde dont le mode ou le degré d'existence conditionne l'adhésion ou la participation du récepteur. En d'autres termes, un document, au sens large, qu'il soit écrit ou audiovisuel, est produit en fonction d'un type de croyance visée par le destinateur et, en retour, il ne peut être interprété par celui qui le reçoit sans une idée préalable du type de lien qui l'unit à la réalité.

Ces mondes sont le monde réel, le monde fictif et le monde ludique. 
3. Loin d'être fixée une fois pour toutes, la place des genres est variable selon le point de vue dont on les considère, et c'est ce qui fait de la communication télévisuelle autre chose qu'une chambre d'enregistrement dans laquelle le récepteur entérinerait la sémantisation des genres par l'émetteur.

$\mathrm{Si}$, comme je le soutiens, la communication télévisuelle est un modèle dynamique où émetteur et téléspectateur ne s'accordent pas toujours sur le sens à donner à un programme, si ces mondes jouent comme des interprétants, au sens peircien, il faut imaginer les genres, non comme des points sur une carte, mais plutôt comme des plaques terrestres susceptibles de déplacements. En sorte que, sur chaque sommet $\mathrm{du}$ triangle, peut être placé un nouveau triangle qui reproduit la configuration de l'ensemble de la carte (à l'image des fractals).

\section{La communication télévisuelle repose sur une double promesse :}

- une promesse ontologique. Cette promesse est contenue dans le nom de genre luimême. Toute « comédie », par exemple, est une promesse de rire, indépendamment de la réussite effective de cette comédie. Toute émission « en direct » est fondée sur la simultanéité de l'événement et de la réception et, du même coup, est porteuse d'une garantie d'authenticité que n'a pas forcément une émission enregistrée. Encore faut-il préciser que cette "promesse », qui rappelle ce que Jauss a pu appeler un « horizon d'attente », n'est pas pour moi un donné. Bien au contraire. Elle fait l'objet d'un savoir plus ou moins bien réparti selon l'âge ou le capital culturel. Un terme apparemment aussi consensuel que « direct " prête à bien des mésinterprétations : les uns l'utilisent pour qualifier des programmes dans lesquels un chanteur chante sur scène sans play-back, d'autres pour désigner des images prises sur le vif (« live »). De même, pour le fameux «contrat de fiction » qui repose sur «the suspension of disbelief »: Eco, qui en fut un de ses zélateurs, a pu observer combien le grand public » faisait fi de ce contrat quand il lisait ses livres (depuis, le Da Vinci Code en a apporté d'autres preuves avec ses queues de touristes qui visitent les lieux du roman...).

- une promesse pragmatique. Cette méconnaissance assez fréquente de ce qu'il faut attendre de tel ou tel genre facilite la seconde promesse, qui, elle, est purement du côté de l'émetteur, comme toute promesse, puisque, comme le rappellent les 
philosophes du langage toute promesse est d'abord unilatérale. Une chose est de savoir ce qu'est le direct ou la fiction, une autre de déterminer si tel ou tel programme est un direct ou une fiction. Comme le le téléspectateur ne sait pas toujours a priori à quel genre une émission se rattache, soit qu'il arrive dans un pays étranger, soit parce qu'il ne connaît pas son "format », il est enclin à croire la catégorisation que lui propose le diffuseur. Pour influer sur les croyances des téléspectateurs, les chaînes attribuent par avance tel nom de genre à telle émission : parce que le « direct » est porteur d'une promesse ontologique d'authenticité, elles n'hésitent pas, nous venons de le voir, à estampiller leurs images de cette mention, même quand c'est faux, ce qui justifie, de la part du téléspectateur, un «droit d'exiger» corrélatif de l'acte promissif.

Ce cadre théorique étant rappelé, j'aimerais le mettre à l'épreuve sur deux fronts : l'évaluation du modèle communicationnel et son opérativité pour un autre objet que la télévision, le cinéma.

\section{Évaluation épistémologique du modèle communicationnel.}

Ayant été élevé au biberon cartésien, dès que je formule une hypothèse, un Malin Génie vient me souffler ses objections à l'oreille, revêtant parfois, pour l'occasion, les habits d'un collègue en chair et en os... «En quoi le modèle de communication que tu proposes, me dit-il, fondé sur un acte d'abord unilatéral et, ensuite seulement, bilatéral, rompt-il avec le paradigme du contrat de lecture, que la plupart des chercheurs acceptent les yeux fermés ?...». Bien que j’aie donné de nombreux arguments, ici où là, pour démontrer cette coupure (notamment Jost 1998), j'en trouve de nouveaux dans les problèmes graphiques que pose la représentation de ce que j'ai appelé supra la tectonique des plaques des mondes télévisuels. Comme on sait peut-être, je représente le terrain de migration des genres télévisuels par un triangle dont chaque sommet représente un monde. Si l'idée paraît claire et distincte (le triangle est l'exemple même de l'idée claire et distincte pour Descartes), dès que l'on veut situer les genres, les idées sont plus confuses. À titre d'exemple heuristique, prenons le monde ludique, dont le territoire est a priori moins clairement délimité et défini que celui des mondes réel ou fictif. Les problèmes 
de délimitation peuvent se poser de deux façons, qui, à la réflexion, font écho aux deux niveaux de promesses que je viens de distinguer.

Qu'est-ce qu'un jeu télévisuel ? Une émission qui repose sur un « contrat ludique » ? Et comment sait-on qu'elle requiert un contrat ludique ? Parce que c'est un jeu... Ce type d'explication nous met dans la situation de la souris qui tourne dans sa roue pour grimper vers une inaccessible cime et qui ne progresse pas d'un centimètre... Partons plutôt de l'idée que tous les jeux n'ont pas le même degré de «ludicité », que leur promesse ontologique diffère. Certains sont éminemment ludiques dans la mesure où ils sont leur propre finalité : ce sont des jeux pour le jeu, comme, par exemple, le saut à l'élastique, la varappe ou le funambulisme, tous ces jeux que Caillois regroupent sous le terme ilinx («tourbillon d'eau » en grec) ou encore les jeux de hasard pur (alea pour Caillois). Depuis Fort Boyard (1998), de nombreuses émissions ont exploité le filon de l'ilinx, de Fear Factor, dans lequel une jeune femme doit supporter de rester quatre minutes dans un aquarium rempli de serpent et de cafards, à Sortez-moi de là, je suis une célébrité (2006) qui joue sur le même ressort.

Les jeux qui sont fondés sur la mimicry (mimétisme en anglais) et qui recouvrent toutes les activités qui simulent ou feignent un personnage sans volonté de tromper le spectateur entretiennent, en revanche, des relations fortes avec les mondes réel et fictif. Ce qu'on appelle télé-réalité, par exemple, est d'abord un jeu de rôle en «grandeur nature »: des candidats doivent atteindre des buts qu'on leur donne, la plupart du temps sous l'autorité d'un maître des jeux (l'animateur) qui leur fixe les épreuves à remplir dans la réalité : former un couple (Loft story), « survivre » sur une île déserte (Survivor), éprouver la fidélité de son compagnon (Temptation Island) ou jouer l'écolier d'un pensionnat avec de « vrais » professeurs et de « vrais » surveillants (Le Pensionnat de Chavagnes). En revanche, quand le jeu consiste à se travestir pour imiter des acteurs, des chanteurs, il se rapproche du monde fictif, puisqu'il s'agit de se glisser dans la peau d'un personnage. C'est le cas de programmes où l'animateur donne un gage à un des participants, qui doivent improviser l'imitation de telle personne célèbre ou jouer une situation (cf. Les Grands enfants, Les Grosses têtes).

La topique des jeux télévisés peut donc être schématisée ainsi : 


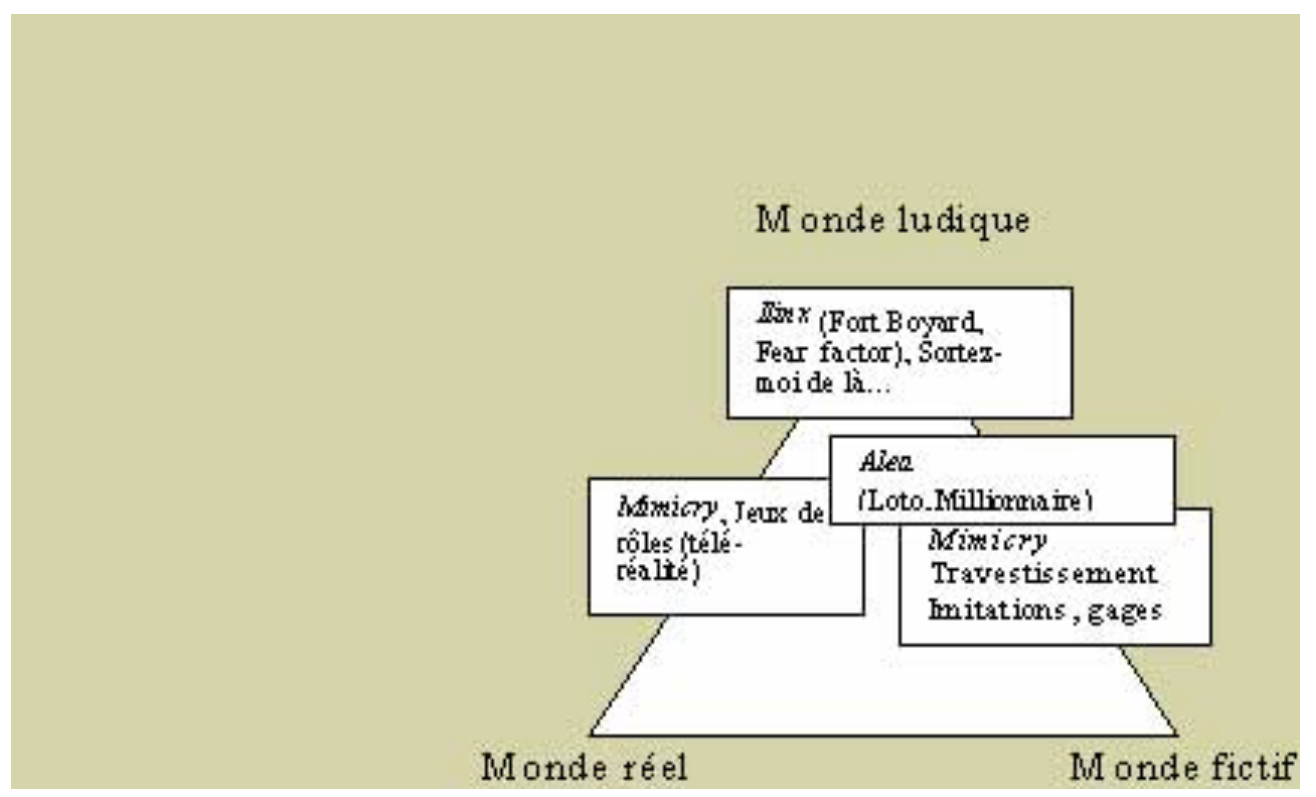

\section{Mapping des jeux télévisés}

Reste à savoir comment situer ce «zoom » sur les jeux sur l'ensemble de la carte des mondes télévisuels. L'aire des jeux est-elle un triangle inscrit, ce qui revient à les placer en fonction de points plus ou moins proches du monde réel ou du monde fictif, comme ceci ?:

Schéma 1

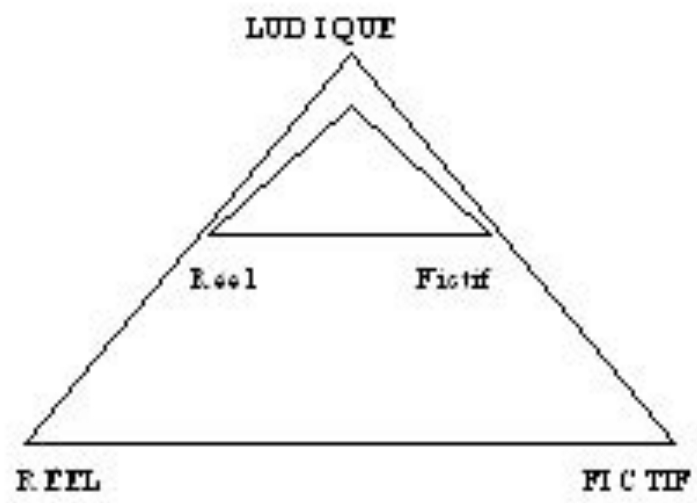


... ou se situe-t-elle hors du premier triangle dans une aire dont le sommet est le monde ludique comme ceci?

Schéma 2

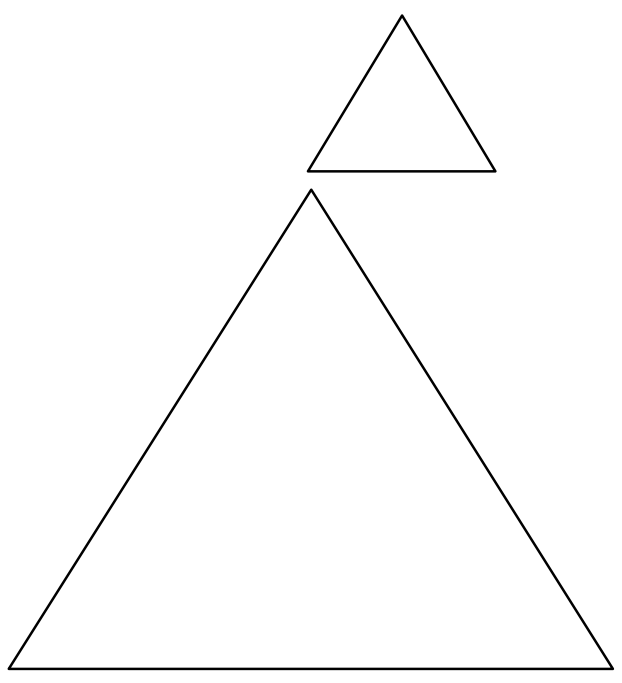

Ces différences de représentation ne sont pas purement formelles, elles engagent en fait deux types de savoirs et de croyances fort différents par rapport au genre du programme que l'on veut situer, comme on le voit dès lors qu'on va chercher la solution dans le second type de promesse, la promesse pragmatique. J'ai admis à l'instant que l'Ile de la Tentation était un jeu. Ce ne fut pourtant pas la promesse de la chaîne, TF1, qui préféra quant à elle ancrer l'émission dans la télé-réalité, mettant l'accent sur le fait que de vrais couples étaient mis à l'épreuve par la confrontation à de «vraies" créatures de rêves... Le fait que trois candidats ont poursuivi les producteurs devant les Prud'hommes, plaidant qu'il ne s'agissait pas d'un jeu mais d'un travail prouve, d'une part, combien l'ancrage générique dans un monde est discutable, mais aussi combien il peut devenir l'enjeu d'un conflit, ce que ne suppose pas évidemment la théorie du contrat, dont le présupposé est la coopération et l'accord des contractants. Les prud'hommes ont donné raison aux candidats, en mars 2006, considérant que l'Ile de la tentation suppose environ 280 heures d'enregistrement. « Les candidats font interview sur interview, en refaisant la prise tant que le producteur n'a pas obtenu ce qu'il souhaite. Ce que voit le téléspectateur n'a rien à voir avec les 
véritables conditions de travail. ${ }^{5} \gg$. L'activité des candidats y est qualifiée de travail dans la mesure où celui-ci est défini comme « la convention par laquelle une personne s'engage à mettre son activité à la disposition d'une autre sous la subordination de laquelle elle se place moyennant rémunération. ${ }^{6}{ }^{\prime}$ Les ex-participants ont touché 1525 euros d'à-valoir sur des contrats de merchandising qui n'ont « abouti à rien ». Enfin les candidats ont été rémunérés. Cette affaire montre combien la catégorisation d'une émission est sujette à caution...

...Autre possibilité : hésiter entre le réel et le jeu, voire le fictif. Ce fut le cas de Pop Stars (Pop Idole) : Fin décembre 2001, la Société des auteurs et compositeurs dramatiques (SACD) et plusieurs institutions défendant des intérêts artistiques et culturels ont engagé devant le Conseil d'État un recours contentieux face à cette « tentation d'élargir la qualification d'œuvre audiovisuelle à des programmes de téléréalité qui ne correspondraient pas aux critères qu'une œuvre exige ». La SACD considérait que l'émission relevait du jeu télévisé, des variétés et de l'autopromotion. Le Conseil d'État a rejeté la requête, considérant qu'elle n'était pas fondée. Il a jugé que Popstars «a pour principal objet de présenter au public l'entraînement, la formation et la progression, dans le domaine de la chanson, des personnes sélectionnées et de décrire un début de carrière effective, au sein des métiers du spectacle », ajoutant que l'émission « comporte des éléments de scénario, une mise en scène et un montage », qui sont des éléments propres à « l'œuvre télévisuelle ». « Le Conseil d'État a relevé que l'émission comportait certes des éléments de jeu et de variétés, mais que ceux-ci ne présentaient qu'un caractère accessoire et n'étaient pas de nature à faire regarder Popstars comme relevant principalement du jeu et des variétés » (Rapport d'activité 2003 du CSA). À terme une telle décision pourrait changer la définition du documentaire. Pour l'instant, je me contenterai de souligner qu'elle relève d'un conflit quant au rattachement de l'émission à un monde, les uns penchant pour le réel, les autres pour le ludique.

\footnotetext{
${ }^{5}$ Voir I. ROBERTS, « La télé-réalité, inconciliable avec la législation sociale », Libération du 22 mars 2006, cité par laurence Leveneur dans son doctorat Histoire et analyse des jeux télévisés.

${ }^{6}$ Ibid.
} 
Comme on voit sur ces exemples, la schématisation 2 (triangle au sommet) et la schématisation 1 (triangle inscrit) ne représentent le même phénomène: la schématisation 2 représente la vision d'un acteur de la communication qui sait que telle ou telle émission relève du jeu, notamment parce qu'il sait ce que jeu veut dire, c'est-à-dire la vision de celui qui a le savoir de ce que tel ou tel génre promet ontologiquement. La notion de contrat, à sa manière (qui n'est pas la mienne) ne pense que cette seule lecture «savante» du programme. Si j'avais pris le cas du monde fictif plutôt que du monde ludique, on pourrait aussi représenter par un triangle extérieur ceux qui, tout en étant au courant du «contrat de fiction » (commandant de suspendre son incrédulité), font des différences entre les fictions « réalistes », les fictions purement fictionnelles (comme celles de la science-fiction ou du merveilleux) et celles qui sont avant tout fondée sur le jeu (celles de tous ces univers qui copient... les jeux-vidéos).

La schématisation 1, en revanche, permet de représenter la relation dynamique qui unit émetteur et récepteur au travers de la promesse pragmatique : le producteur ou le diffuseur tire le programme vers tel monde, par leur étiquetage générique, par leur communication ou leurs publicités et le récepteur accepte plus ou moins cette position de l'émission sur la carte des mondes selon sa crédulité ou sa capacité d'analyse. Dans un cas (triangle inscrit), il n'y a pas d'ambiguïté sur le statut ludique du programme, mais simplement sur le degré de ludicité qu'il contient ; dans l'autre, l'enjeu de la catégorisation est de savoir si le programme réfère à la réalité ou au monde ludique.

\section{Des genres télévisuels aux genres cinématographiques}

On peut se demander à présent si ce modèle dynamique de la communication télévisuel, sur un terrain délimité par un triangle, est lié au fait que la télévision est par définition un lieu d'incertitude, où on ne sait pas toujours à l'avance quels genres on va rencontrer, ou si, au contraire, il est aussi opératoire pour le cinéma. 
Une chose est sûre : jusqu'à une date récente, il n'était pas d'usage d'aborder les films par le biais de grands ensembles comme le genre 7 (Moine) et peu de chercheurs, hormis Roger Odin, se réclamaient de l'approche communicationnelle, pour des raisons institutionnelles qui ont été explicitées en introduction. Pourtant, j'ai pu montrer ailleurs que la séance de cinéma dans un grand théâtre comme l'Hippodrome, au début du siècle, avait tous les caractères de la sérialité télévisuelle, mixte de programmation horizontale et verticale : à heure presque fixe, le programme, comme une grille, venait apporter les émotions que tel ou tel genre promettait au spectateur, et ces émotions étaient réglées aussi par trois mondes : le réel, avec ses « actualités » et ses documents, le fictifs, avec ses mélos, et le ludique, avec ses burlesques et ses attractions sur scène (Jost in Quaresima 1999).

Et aujourd'hui, une telle approche via les archigenres est-elle productive?

Pour répondre à cette question centrale, on peut partir de l'opposition documentaire/fiction. Quel est son «savoir tacite», comme disent aujourd'hui certains épistémologues? À n'en pas douter que ce sont les critères matériels des films qui les opposent et qu'il est possible de faire la différence entre un documentaire et une fiction à partir d'indices ou de procédés codiques. En cela, cette dichotomie est tout à fait solidaire des présupposés de la sémiologie du cinéma de première génération. Or, si l'on considère un film comme Blair Witch Project, par exemple, on constate immédiatement que cette façon de façon voir ne nous aide en rien. Voici un film, qui a été présenté au monde entier par internet, bien avant sa sortie, comme un documentaire sur une bande de jeunes qui part à la recherche de sorcière dans la forêt de Blair et dont des caméras retrouvées près de leurs cadavres avec les films a permis de retracer l'odyssée. Du point de vue du «bon » spectateur qui entra dans les «consignes» (Odin) données par la bande-annonce ou le générique, l'intentio lectoris a visé Blair witch Project comme un documentaire. Pour l'analyste en revanche, qui sait que ce film a été joué, parfaitement d'ailleurs, par des acteurs, il relève au contraire des critères définitoires de la fiction, si l'on admet, à la suite de K. Hamburger, que la fiction ne se définit pas par comparaison avec la

7 Cela a été réparé par divers ouvrages: La nascita dei generi cinematografici/the Birth of Film genres, Udine, Forum, 1999, R. Altamn, Film/Genre, Londres, BF1, 1999, Raphaëlle Moine, Les Genres du cinéma, Nathan, 2002. 
réalité, mais par le seul fait qu'elle s'ancre dans des Je-Origines fictifs. Du point de vue communicationnel, cet exemple confirme que le cinéma à l'ère du marketing ne fonctionne pas sur un autre modèle que la télévision. La communication médiatique se moque de la sincérité que suppose le contrat et préfère promettre un sens qui divise forcément les spectateurs en deux catégories, les crédules et les sceptiques. Ce fonctionnement est d'autant plus prégnant dans le cinéma d'aujourd'hui que tout film, pour exister, doit faire avec sa diffusion télévisuelle, faute d'obtenir un financement.

Si la promesse est première dans la communication cinématographique comme dans le communication télévisuelle, il semble que les enjeux sémantiques ne soient guère différents pour les programmes lors de leur première diffusion et pour les films au moment de leur sortie. Le cas de Blair Witch s'est ainsi généralisé. Si tous les films ne prétendent pas être des documentaires, le lancement de beaucoup d'entre eux se fonde sur une négation du «contrat de fiction ». L'argument principal qui commande au lecteur de croire - et non pas de suspendre son incrédulité - est le fait que le film est «basé sur des faits réels »: de la Passion du Christ, que certains hésitent à considérer comme une fiction, étant donné la noblesse du sujet, à $D a$ Vinci Code qui prétend nous révéler la vraie histoire de Jésus, en passant par les derniers jours d'Hitler, dans La Chute, les films de fictions, c'est-à-dire, pour aller vite, comme je viens de le dire, ceux qui s'appuient sur un Je-Origine fictif, un acteur, prétendent de plus en plus raconter au nom du réel, c'est-à-dire, tout bonnement montrer. Voulant me changer les idées dix minutes, je tombe sur un article du Monde, qui présente un film de Cannes 2006 par ces mots : «le scénario de Buenos Aires 1977 est inspiré d'une histoire vraie» (Le Monde, 30 mai 2006). Évidemment tout ce mouvement d'authentification de la fiction a pour pendant l'avènement du « docufiction » à la télévision, films qui prétendent parler du réel, mais qui le font avec des acteurs.

Le monde ludique est-il un interprétant qui s'impose pour le cinéma comme pour la télévision ? Sans doute moins, dans la mesure où le cinéma ne connaît que la forme «Film » et non les divertissements en plateau qui sont le terreau privilégié du ludique télévisuel. Néanmoins, le cinéma, dès ses débuts, trouve ses racines dans le jeu. Méliès, le premier sans doute, a affirmé cette prééminence du cinéma-comme-jeu 
sur le cinéma de fiction, à propos de ses scénarios : « J'ai fait, durant vingt ans, des films fantastiques de tous genres, et ma première préoccupation était de trouver, pour chaque film, des trucs inédits, un grand effet principal et une apothéose finale. Après cela [mes italiques], je cherchais quelle époque serait la meilleure pour habiller mes personnages (...). Quant au scénario, à la 'fable', au 'conte', je m'en occupais en dernier. Je puis affirmer que le scénario ainsi fait n'avait aucune importance... ${ }^{8}$. De la même façon, et à peu près à la même époque, le film de poursuite s'appuyait sur une structure narrative connue d'avance et son intérêt ne résidait pour le spectateur que dans les attractions qui scandaient la poursuite. Cet aspect éminemment ludique du cinéma a été ravivé au tournant du siècle par les films catastrophes ou les films à effets spéciaux, dont les situations paroxystiques nous retiennent moins pour leur suspens que pour les sensations qu'ils suscitent, proches de ces jeux d'ilinx, que Caillois définissait comme la recherche ludique du vertige. Certains comparent d'ailleurs à juste titre certains films des années 90 à des tours de manège (Jullier 1997).

En se déplaçant vers le paradigme communicationnel et les médias, la sémiologie du cinéma s'est trouvée bouleversée à tous les niveaux : celui de l'objet $d u$ signe : non plus le monde, mais la source, non plus un objet langagier indifférencié, mais des médias; celui de la méthode, qui a opéré un véritable retournement copernicien : au lieu de partir de l'image pour aller vers de plus grands ensembles textuels, les seconds sont devenus premiers. Du même coup, c'est le modèle communicationnel qui s'en est trouvé modifié. Il faut aujourd'hui aller plus loin et se demander ce que change, pour tous les objets culturels, -livres, films, émissions ou CD - d'être considérés d'abord comme des médias.

\footnotetext{
${ }^{8}$ Cité par Sadoul, Méliès, Paris, Seghers, p. 115-116. 


\section{Ouvrages cités :}

ALTMAN RICK, Film/Genre, Londres, BF1, 1999

BARTHES ROLAND, Critique et vérité, Seuil

CHARAUDEAU PATRICK [1997], Le Discours d'information médiatique, Nathan

DANTO ARTHUR, La Transfiguration du banal,

HAMBURGER KÄTE ([1957] 1986), Logique des genres littéraires, trad. fr., Seuil

HARA YOMIKO, TOMOMUNE YOMIKO et SHIGEMORI MAKI [2004],

" Categorization of Japonese Viewers based on Program Genres they watch », in User Modeling and User-adaptated Interaction $14: 87-117$, Pays-Bas, Kluwer Academic Publishers.

JOST FRANÇOIS [1992], Un Monde à notre image. Enonciation, cinéma, Télévision, Méridiens Klincksieck

JOST FRANÇOIS [1998]

JOST FRANÇOIS [1998], « Le genre télévisuel : du contrat au genre », Degrés nº94.

METZ CHRISTIAN,- [1968], Essais sur la signification au cinéma, Klincksieck, 1968

MOINE RAPHAËLLE, Les Genres du cinéma, Nathan, 2002.

QUARESIMA LEONARDO, RAENGO ALESSANDRA, VICHI LAURA [1999], La nascita dei generi cinematografici/the Birth of Film genres, Udine, Forum, 1999

SPERBER DAN et WILSON DEIRDRE , La Pertinence, Minuit 